PREPARED FOR THE U.S. DEPARTMENT OF ENERGY, UNDER CONTRACT DE-AC02-76CH03073

PPPL-3692

PPPL-3692

UC-70

Energy of Force-free Magnetic Fields in Relation to Coronal Mass Ejections

by

G.S. Choe and C.Z. Cheng

May 2002

M

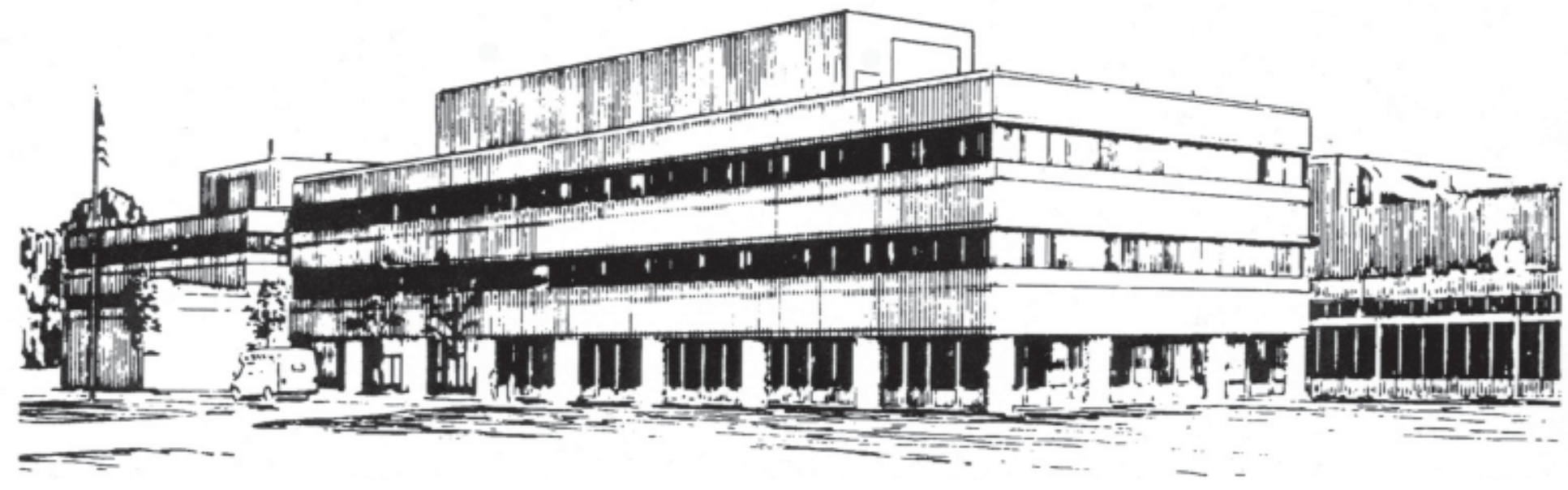

PRINCETON PLASMA PHYSICS LABORATORY PRINCETON UNIVERSITY, PRINCETON, NEW JERSEY 


\section{PPPL Reports Disclaimer}

This report was prepared as an account of work sponsored by an agency of the United States Government. Neither the United States Government nor any agency thereof, nor any of their employees, makes any warranty, express or implied, or assumes any legal liability or responsibility for the accuracy, completeness, or usefulness of any information, apparatus, product, or process disclosed, or represents that its use would not infringe privately owned rights. Reference herein to any specific commercial product, process, or service by trade name, trademark, manufacturer, or otherwise, does not necessarily constitute or imply its endorsement, recommendation, or favoring by the United States Government or any agency thereof. The views and opinions of authors expressed herein do not necessarily state or reflect those of the United States Government or any agency thereof.

\section{Availability}

This report is posted on the U.S. Department of Energy's Princeton Plasma Physics Laboratory Publications and Reports web site in Fiscal Year 2002. The home page for PPPL Reports and Publications is: http://www.pppl.gov/pub_report/

DOE and DOE Contractors can obtain copies of this report from:

U.S. Department of Energy

Office of Scientific and Technical Information

DOE Technical Information Services (DTIS)

P.O. Box 62

Oak Ridge, TN 37831

Telephone: (865) 576-8401

Fax: (865) 576-5728

Email: reports@adonis.osti.gov

This report is available to the general public from:

National Technical Information Service

U.S. Department of Commerce

5285 Port Royal Road

Springfield, VA 22161

Telephone: 1-800-553-6847 or

(703) 605-6000

Fax: (703) 321-8547

Internet: http://www.ntis.gov/ordering.htm 
Submitted to Ap.J.

\title{
Energy of Force-Free Magnetic Fields in Relation to Coronal Mass Ejections
}

\author{
G. S. Choe and C. Z. Cheng \\ Princeton Plasma Physics Laboratory, Princeton University, Princeton, NJ 08543-0451 \\ gchoe@pppl.gov
}

\begin{abstract}
In typical observations of coronal mass ejections (CMEs), a magnetic structure of a helmet-shaped closed configuration bulges out and eventually opens up. However, a spontaneous transition between these field configurations has been regarded to be energetically impossible in force-free fields according to the AlySturrock theorem. The theorem states that the maximum energy state of forcefree fields with a given boundary normal field distribution is the open field. The theorem implicitly assumes the existence of the maximum energy state, which may not be taken for granted. In this study, we have constructed force-free fields containing tangential discontinuities in multiple flux systems. These force-free fields can be generated from a potential field by footpoint motions that do not conserve the boundary normal field distribution. Some of these force-free fields are found to have more magnetic energy than the corresponding open fields. The constructed force-free configurations are compared with observational features of CME-bearing active regions. Possible mechanisms of CMEs are also discussed.
\end{abstract}

Subject headings: Sun: coronal mass ejections (CMEs) - magnetic fields - magnetohydrodynamics (MHD)

\section{Introduction}

A typical sequence of coronal mass ejection ( $\mathrm{CME}$ ) consists of rising of a cavity and formation of a CME loop, opening up of field lines, and magnetic reconnection of open fields manifested as a flare, which recovers the closed field configuration as before the eruption (e.g., Hundhausen 1988, 1999; Gosling 1993a,b). These sequential processes are considered 
to occur spontaneously because the timescale of a CME (hours to a day) is much shorter than the timescale of energy buildup (days to a month). The free energy driving a CME is believed to be stored in the pre-eruption magnetic field, most part of which has a closed configuration. Here the term 'closed' means that both ends of field lines are connected to the solar surface. A spontaneous transition from a closed magnetic configuration to an open field configuration requires that more magnetic energy should be contained in the closed magnetic field than in the open field.

The investigation of this energy hypothesis dates back to early days of flare research (Barnes \& Sturrock 1972). Due to the dominance of magnetic field pressure over plasma pressure in the corona, the research has been mostly concentrated on force-free fields (for brevity hereafter FFFs). A ground-breaking progress in this research was made by Aly (1984) who showed that there is an upper bound of magnetic energy of force-free fields in an infinite halfspace with the same boundary normal field (hereafter $B_{n}$ ) distribution. Aly (1984) further conjectured that the maximum energy state of the closed FFFs should be the corresponding open field. This conjecture was backed up by "physical proofs" provided by Aly (1991) and Sturrock (1991) and since then has been commonly called "Aly-Sturrock theorem."

As Aly (1991) pointed out, the proofs by Aly (1991) and Sturrock (1991) are not complete because their validity is conditioned by two important assumptions. First, in the set of admissible FFFs, whose lines are connected to the bottom boundary, an energy maximizing sequence of FFFs should be able to converge to a field $\boldsymbol{B}+$, which may or may not belong to the set. Second, if the sequence converges, it should hold that $E[\boldsymbol{B}+]=E_{m}$, where $E[\boldsymbol{B}+]$ designates the magnetic energy of $\boldsymbol{B}+$ and $E_{m}$ is the least upper bound of energy of the FFFs. Although these two conditions intuitively seem trivial, their validity cannot be taken for granted at all. Even if $\boldsymbol{B}+$ exists, there is a possibility that $E[\boldsymbol{B}+]<E_{m}$ (J. J. Aly 2002, private communication). In other words, no admissible FFF configuration may exist for the energy supremum $E_{m}$. The argument in $\S 4$ of Sturrock (1991) does not consider such a possibility. In short, no rigorous proof for Aly's conjecture has been presented yet.

Aly's conjecture has been supported by quite a few numerical (Yang et al. 1986; Mikić \& Linker 1994; Roumeliotis et al. 1994; Amari et al. 1996) and analytical (Lynden-Bell \& Boily 1994; Aly 1994a; Wolfson 1995) studies of twisted or sheared magnetic fields. All these studies dealt with only those force-free states which are physically accessible under the ideal MHD conditions from potential fields by footpoint motions always conserving the boundary normal field distribution. In this Letter, we investigate force-free fields numerically constructed in multiple flux systems. The FFFs in our study differ from the FFFs in the previous studies in that our FFFs contain singular current sheets (tangential discontinuities) 
and that our FFFs cannot be generated from a potential field by $B_{n}$-conserving footpoint motions. We find that some of our FFFs have more energy than the corresponding open fields. In $\S 2$, we describe our FFF model and the numerical procedure. Our computational results for field configurations and magnetic energy are presented in $\S 3$. In $\S 4$, a comparison is made between our results and observational features of eruptive active regions. A summary and discussions regarding possible mechanisms of CMEs are provided in $§ 5$.

\section{Modeling of Force-Free Fields in Two-Flux Systems}

Our study of multiple flux systems is motivated by the fact that magnetic fields in and below the solar photosphere are made of filamentary flux tubes. After a magnetic field emerges from below the solar surface, the high $\beta$ plasma barrier between elementary flux tubes will be drained and only current sheets will separate individual flux tubes. Unless magnetic reconnection totally destroys the current sheets, the magnetic field will probably retains the cellular structure. The simplest model of such a cellular magnetic structure is two interwinding flux tubes. Formation of interwinding flux tubes of a much larger scale is expected when a new flux tube emerges under a pre-existing field. Since there is no reason for these two flux systems to have any connectivity, magnetic field is discontinuous in the interface of two flux systems. Large scale photospheric motions can interwind these flux tubes.

We consider a magnetic field system occupying the infinite halfspace $\{z>0\}$ above a plane. The magnetic field normal to the boundary plane $\{z=0\}$ is concentrated on four separate circular patches of finite area so that

$$
B_{n}(x, y)=B_{z}(x, y, 0)=\sum_{i} B_{z i}\left(\rho_{i}\right)
$$

where

$$
B_{z i}\left(\rho_{i}\right)= \begin{cases} \pm\left[1-\left(\rho_{i} / R\right)^{2}\right]^{2} & \text { if } \rho_{i} \leq R \\ 0 & \text { if } \rho_{i}>R\end{cases}
$$

in which $\rho_{i}=\left|\boldsymbol{r}-\boldsymbol{r}_{i}\right|$ is the distance from the center position of the $i$-indexed patch, $\boldsymbol{r}_{i}$, to a point $\boldsymbol{r}$ in the $z=0$ plane and $R$ is the radius of the patches. We assume that for $x_{i}>0$, $B_{z i}>0$, and for $x_{i}<0, B_{z i}<0$. Let's consider two flux tubes designated by $a$ and $b$, whose intersections with the $z=0$ plane are circular patches of radius $R=0.7$ centered at

$$
\begin{array}{ll}
\boldsymbol{r}_{a+}=(1.7,0,0), & \boldsymbol{r}_{a-}=(-1.7,0,0) \\
\boldsymbol{r}_{b+}=(3.3,0,0), & \boldsymbol{r}_{b-}=(-3.3,0,0)
\end{array}
$$


One possible configuration of the two-flux system (not in equilibrium) satisfying the above boundary conditions is shown in Figure 1(a). The potential field satisfying the boundary condition given by equations (1)-(3) will have the field line connectivity as follows:

$$
x_{o+}=-x_{o-}, \quad y_{o+}=y_{o-},
$$

where $\left(x_{o+}, y_{o+}\right)$ and $\left(x_{o-}, y_{o_{-}}\right)$are coordinates of two conjugate field line footpoints, one in $x>0$ and the other in $x<0$. Now we impose horizontal boundary motions respectively on each side of the polarity inversion line so that each pair of flux patches undergoes a rotational motion with a constant angular velocity centered at $\boldsymbol{r}_{\mathrm{C} \pm}=\left(\boldsymbol{r}_{a \pm}+\boldsymbol{r}_{b \pm}\right) / 2=( \pm 2.5,0)$. Beyond a distance wholly covering each pair of flux patches, the angular speed is assumed to be gradually tapered down to 0 with the increasing distance. Under ideal MHD condition, two flux tubes are interwound by this rotational boundary motion, conserving the field line connectivity. The angle of twist (or interwinding) between two flux tubes, which will be denoted by $\Phi$, is twice the rotation angle on each side of the polarity inversion line. Figure 1(b) shows one possible configuration of the two-flux system (not in equilibrium) for $\Phi=2 \pi$. If we relax the two-flux system to a force-free equilibrium after interwinding by any nonzero angle $\Phi$, part of the separatrix between two flux systems will become a current sheet (tangential discontinuity). The field connectivity of a FFF with $\Phi \neq 0$ is definitely different from that of the potential field for the same $B_{n}(x, y)$ and cannot be created from the potential field by any footpoint motions conserving $B_{n}(x, y)$ under the ideal MHD conditions.

In this study, we have numerically constructed FFFs for different twist angles. Since our FFFs contain current sheets, it is important to prevent any spurious magnetic reconnection and maintain the specified field connectivity. To do this, we describe the magnetic field with 'unmatched Euler potentials' (e.g, Stern 1970) as

$$
\boldsymbol{B}=\sum_{j} F_{j}\left(\alpha_{j}, \beta_{j}\right)\left(\nabla \alpha_{j} \times \nabla \beta_{j}\right),
$$

where index $j$ denotes the flux system $a$ or $b$. Either $F_{a}$ or $F_{b}$ is only allowed to have a nonzero value at any location $(x, y, z)$. The Euler potential description has the advantage of inducing far less spurious magnetic reconnection than employing the three components of a magnetic field $\boldsymbol{B}$ or a vector potential $\boldsymbol{A}$. To specify the location of field line footpoints easily, we select a functional form of Euler potentials as

$$
\alpha=\alpha\left(x_{o}^{2}\right), \quad \beta=\beta\left(y_{o}\right),
$$

where $x_{o}$ and $y_{o}$ are the coordinates of field line footpoints at $z=0$ when the angle of twist $\Phi$ is zero. With this setting, we have

$$
B_{z}(x, y, 0)=\sum_{j} F_{j}\left(\alpha_{j}, \beta_{j}\right)\left(\frac{d \alpha_{j}}{d x_{o}}\right)\left(\frac{d \beta_{j}}{d y_{o}}\right) .
$$


With the field description given by equation (5), the magnetic induction equation (Faraday's law) can simply be written as

$$
\frac{\partial \alpha}{\partial t}=-\boldsymbol{v} \cdot \nabla \alpha, \quad \frac{\partial \beta}{\partial t}=-\boldsymbol{v} \cdot \nabla \beta,
$$

where $\boldsymbol{v}$ is the bulk velocity of plasma. To construct force-free equilibria, we have employed a magnetofrictional method (e.g., Chodura \& Schlüter 1981; Choe \& Lee 1996), which drives the system to the minimum energy state by continuously removing kinetic energy with the field connectivity conserved. In this method, we also employ equation (8), in which $t$ is now a relaxation parameter and $\boldsymbol{v}$ is a vector proportional to the Lorentz force.

In specifying boundary conditions, we have to consider two points. First, different from time-dependent problems (e.g., Mikić \& Linker 1994; Amari et al. 1996), an equilibrium problem is well defined only if $B_{n}$ is specified at all boundaries (Chodura \& Schlüter 1981). Second, the stress exerted by the boundary should be minimized in order to simulate an open system. Thus, we specify $B_{n}$ as given by equations (1)-(3) at the bottom boundary and set $B_{n}$ to zero at all other boundaries. This boundary condition naturally allows field movement tangential to the boundary where $B_{n}=0$ during the relaxation process.

\section{Constructed Force-Free Fields and Their Energy}

We have constructed a total of seven force-free fields with $\Phi=0,0.5 \pi, \pi, 1.5 \pi, 2 \pi$, $2.5 \pi$ and $3 \pi$. Two flux surfaces containing a quarter of the total flux in each flux tube are shown in Figure 2 for $\Phi=1.5 \pi$ and $3 \pi$. It is interesting that the flux system occupying a finite flux volume at $\Phi=0$ (the red one in Figures 1 and 2) always takes a finite volume after interwinding whereas the other system (the green one) always takes an infinite volume (the rest volume). The notable pleated structure of the flux surfaces can be attributed to the self-twist of the flux tubes generated by the rotational footpoint motion.

The magnetic energy of the force-free fields that we have constructed increases with the twist angle as shown in Figure 3. The energies of the potential fields and of the open fields can be obtained with a Green function method familiar in electrostatics and are plotted in the figure for reference. An upper bound (not the least upper bound) of energy of FFFs derived by Aly (1984),

$$
E_{U B}=\frac{1}{4 \pi}\left(\int_{z=0} B_{n}^{2} d x d y\right)^{\frac{1}{2}}\left(\int_{z=0} r^{2} B_{n}^{2} d x d y\right)^{\frac{1}{2}},
$$

is $10.7 E_{\text {pot }}(\Phi=0)=8.69 E_{\text {open }}(\Phi=0)$, far greater than the energies we have obtained. In the figure, we can find that the magnetic energy of FFFs exceeds the open field energy for $\Phi \gtrsim 1.5 \pi$. 
Although we deal with systems in an infinite halfspace, our computation is performed in a finite computational box. Thus, we need to make sure that the uncertainty in energy does not affect our conclusion. To do this, we first compared the energies of force-free fields that we obtained in domains of different sizes. The energies obtained in a box of $160 \times 160 \times 160$ (refer eq. [3] for units) differ from those obtained in a box of the size $300 \times 300 \times 300$ by not more than $5 \%$. The latter differ from the energies obtained in a box of the size $400 \times 400 \times 400$ by not more than $1.4 \%$. Furthermore, the last two configurations can hardly be distinguished. The values we presented are obtained with the largest box. Another way of assessing the effect of the finite box size is to check integral relations that should be satisfied by force-free fields in an infinite halfspace above a plane. Aly (1984) derived the following equations from the tensor virial theorem.

$$
\begin{gathered}
\int_{z}\left(B_{x}^{2}+B_{y}^{2}\right) d x d y=\int_{z} B_{z}^{2} d x d y . \\
E(z>0)=\frac{1}{8 \pi} \int_{z>0}\left(B_{x}^{2}+B_{y}^{2}+B_{z}^{2}\right) d x d y d z=\frac{1}{4 \pi} \int_{z=0}\left(x B_{x}+y B_{y}\right) B_{z} d x d y .
\end{gathered}
$$

For our FFF solutions, the discrepancy between the lefthand side and the righthand side of the above equations is found to be less than $3 \%$ for equation (9) and of less than $9 \%$ for equation (10), respectively. For not too large twist angles, the discrepancies are even smaller than the above values. The energy of the constructed force-free field for $\Phi=3 \pi$ is about $30 \%$ more than the open field energy. This difference is considered to be well above the error level due to the finite size of the domain. Therefore, we can conclude that some of the force-free fields we have constructed indeed have more energy than the open field.

What, then, makes our results on multiple flux systems different from the results of previous studies on single flux systems (Yang et al. 1986; Mikić \& Linker 1994; Roumeliotis et al. 1994; Amari et al. 1996; Lynden-Bell \& Boily 1994; Aly 1994a; Wolfson 1995)? The single flux systems treated in most previous studies (except Lynden-Bell \& Boily 1994) have smooth, well-ordered field structures. Their field lines tend to partially open beyond a certain shear or twist so that further accumulation of magnetic energy in the system is hindered. On the contrary, we do not find any tendency of field opening in our interwinding two-flux system although the system takes a larger flux volume with increasing $\Phi$. By interwinding, each flux system seems to suppress free expansion of the other. This shackling behavior of twisted fields is also reported by Klimchuk et al. (2000) for a single flux system in a smooth equilibrium. 


\section{Relevance to Observations}

The most well-known observational condition for solar eruption detected in the photosphere and chromosphere is the high magnetic shear (e.g., Krall et al. 1982), which is the alignment of horizontal magnetic field vectors along the polarity inversion line. In addition, polarity inversion lines also show a tendency of distortion during the evolution of active regions toward eruption (e.g., Uddin et al. 1986). Recent X-ray observations have revealed appearance of an S- or inverse-S-shaped bundle of coronal loops before solar eruption (Acton et al. 1992; Canfield et al. 1999). This structure is called a sigmoid (Rust \& Kumar 1996).

To compare our force-free field model with these observations, we have generated vectormagnetograms at a height level of $z=0.22$ with our FFF solutions. Also images projected onto the bottom boundary are created for a flux surface containing $95 \%$ of the total flux of the inner flux tube. Figure 4 shows one set (for $\Phi=2.5 \pi$ ) of those plots. As much expected, the magnetic shear increases along the polarity inversion line with the twist angle. Moreover, the polarity inversion line becomes more tilted and distorted from the original straight line

with the increase of the twist angle. The projected image of a flux surface takes an inverse-S shape and becomes more twisted and larger in size with increasing $\Phi$. We note that the outermost flux surface of the inner flux tube, which is the separatrix between the two flux systems, also takes a shape similar to the flux surface shown in the figure, but with a little larger scale. If the emission from a sigmoid is due to the heating by magnetic reconnection in the pre-eruption stage, the resulting change in field topology may play an important role in the subsequent solar eruption.

\section{Summary and Discussion}

We have found that there is a class of force-free fields of closed field configuration having more energy than the open fields. The force-free fields considered in our study consist of two interwinding flux systems with current sheets.

Although we have constructed closed force-free fields having more energy than the open fields, we still do not know whether they can lead to a CME eruption and what kind of mechanism is involved if eruption can take place. For future studies, we can think of several possibilities leading to eruption. The eruption up to field opening may be solely an ideal MHD process or may somehow involve magnetic reconnection. In the former case, a global nonequilibrium (see Aly 1994b, for the definition) may take place beyond a certain amount of twist. However, our study so far does not hint this possibility. If magnetic reconnection is involved, a variety of possibilities can arise. Magnetic reconnection results in change 
of field topology. If no equilibrium of closed configuration is available in a field topology created during a reconnection process and if the field still retains more energy than the open field, an eruption with field opening can take place. If closed equilibrium states are always available in field topologies generated by a reconnection process and if flux volumes become much larger in the new equilibrium states, CMEs with apparent field opening to a finite distance can occur. If flux volumes in the new equilibrium states are not considerably larger than the original flux volume, a flare may take place without a CME. Investigation of these possibilities will be performed in our future studies.

We greatly thank Dr. J.-J. Aly for helpful comments and discussions. This work has

been supported by DoE Contract No. DE-AC02-76-CH03073 and NSF grant ATM-9906142.

\section{REFERENCES}

Acton, L., et al. 1992, Science, 258, 618

Aly, J. J. 1984, ApJ, 283, 349

Aly, J. J. 1991, ApJ, 375, L61

Aly, J. J. 1994a, A\&A, 288, 1012

Aly, J. J. 1994b, in Cosmical Magnetism, Contributed Papers of the NATO Advanced Research Workshop, ed. D. Lynden-Bell (Cambridge: Institute of Astronomy), 7

Amari, T., Luciani, J. F., Aly, J. J., \& Tagger, M. 1996, ApJ, 466, L39

Barnes, C. W., \& Sturrock, P. A. 1972, ApJ, 174, 659

Canfield, R. C., Hudson, H. S., \& McKenzie, D. E. 1999, Geophys. Res. Lett., 26, 627

Chodura, R., \& Schlüter, A. 1981, J. Comp. Phys., 41, 68

Choe, G. S., \& Lee, L. C. 1996, ApJ, 472, 360

Gosling, J. T. 1993a, Physics of Fluids B, 5, 2638

Gosling, J. T. 1993b, J. Geophys. Res., 98, 18937

Hundhausen, A. J. 1988, in Proceedings of the Sixth International Solar Wind Conference, ed. V. J. Pizzo, T. E. Holzer, \& D. G. Sime (Boulder: NCAR Technical Note 306), 181 
Hundhausen, A. J. 1999, in The Many Faces of the Sun: A Summary of the Results from NASA's Solar Maximum Mission, ed. K. T. Strong, J. L. R. Saba, B. M. Haisch, \& J. T. Schmelz (New York: Springer-Verlag), 143

Klimchuk, J. A., Antiochos, S. K., \& Norton, D. 2000, ApJ, 542, 504

Krall, K. R., Smith, J. B., Hagyard, M. J., West, E. A., \& Cummings, N. P. 1982, Sol. Phys., 79,59

Lynden-Bell, D., \& Boily, C. 1994, MNRAS, 267, 146

Mikić, Z., \& Linker, J. A. 1994, ApJ, 430, 898

Roumeliotis, G., Sturrock, P. A., \& Antiochos, S. K. 1994, ApJ, 423, 847

Rust, D. M., \& Kumar, A. 1996, ApJ, 464, L199

Stern, D. P. 1970, Amer. J. Phys., 38, 494

Sturrock, P. A. 1991, ApJ, 380, 655

Uddin, W., Pande, M. C., \& Shelke, R. N. 1986, Bulletin of the Astronomical Society of India, 14, 91

Wolfson, R. 1995, ApJ, 443, 810

Yang, W. H., Sturrock, P. A., \& Antiochos, S. K. 1986, ApJ, 309, 383 
(a) $\Phi=0$

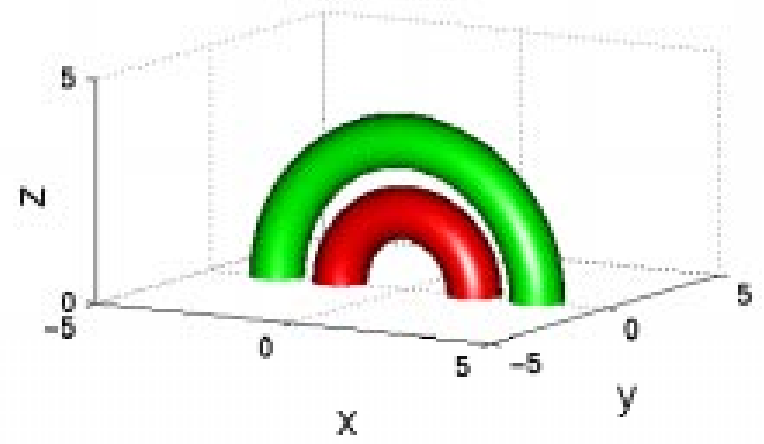

(b) $\Phi=2 \pi$

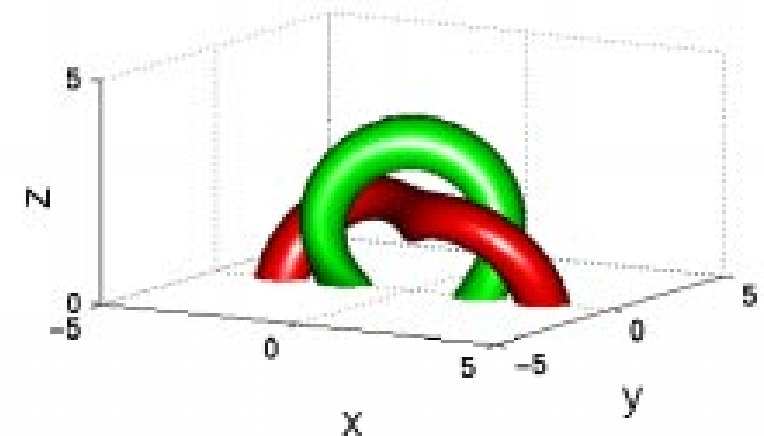

Fig. 1.- Possible configurations of a two-flux system (not in equilibrium). The configuration in (a) has the same field connectivity as the potential field. The configuration in (b) can be created by rotational boundary motions under the ideal MHD conditions.

(a) $\Phi=1.5 \pi$

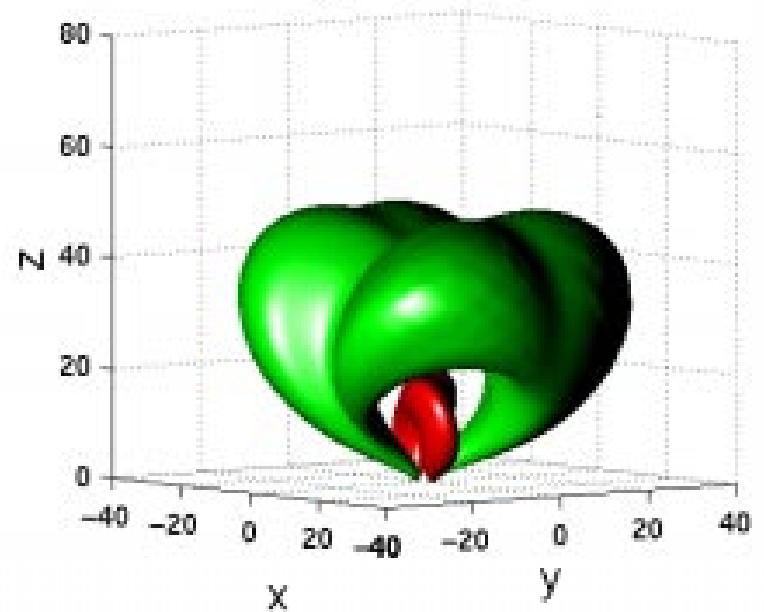

(b) $\Phi=3 \pi$

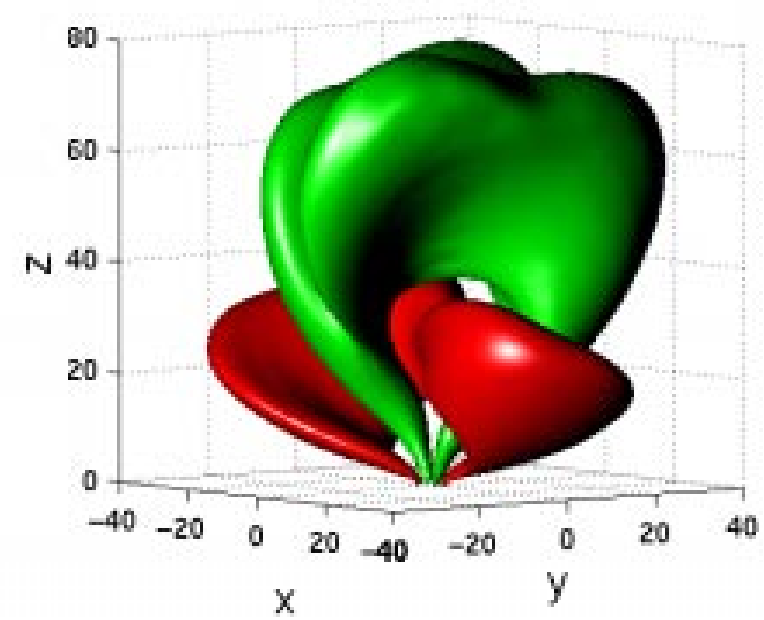

Fig. 2.- Flux surfaces of the numerically generated force-free fields consisting of two interwinding flux systems. Each flux surface contains $25 \%$ of the total flux in each flux system. 


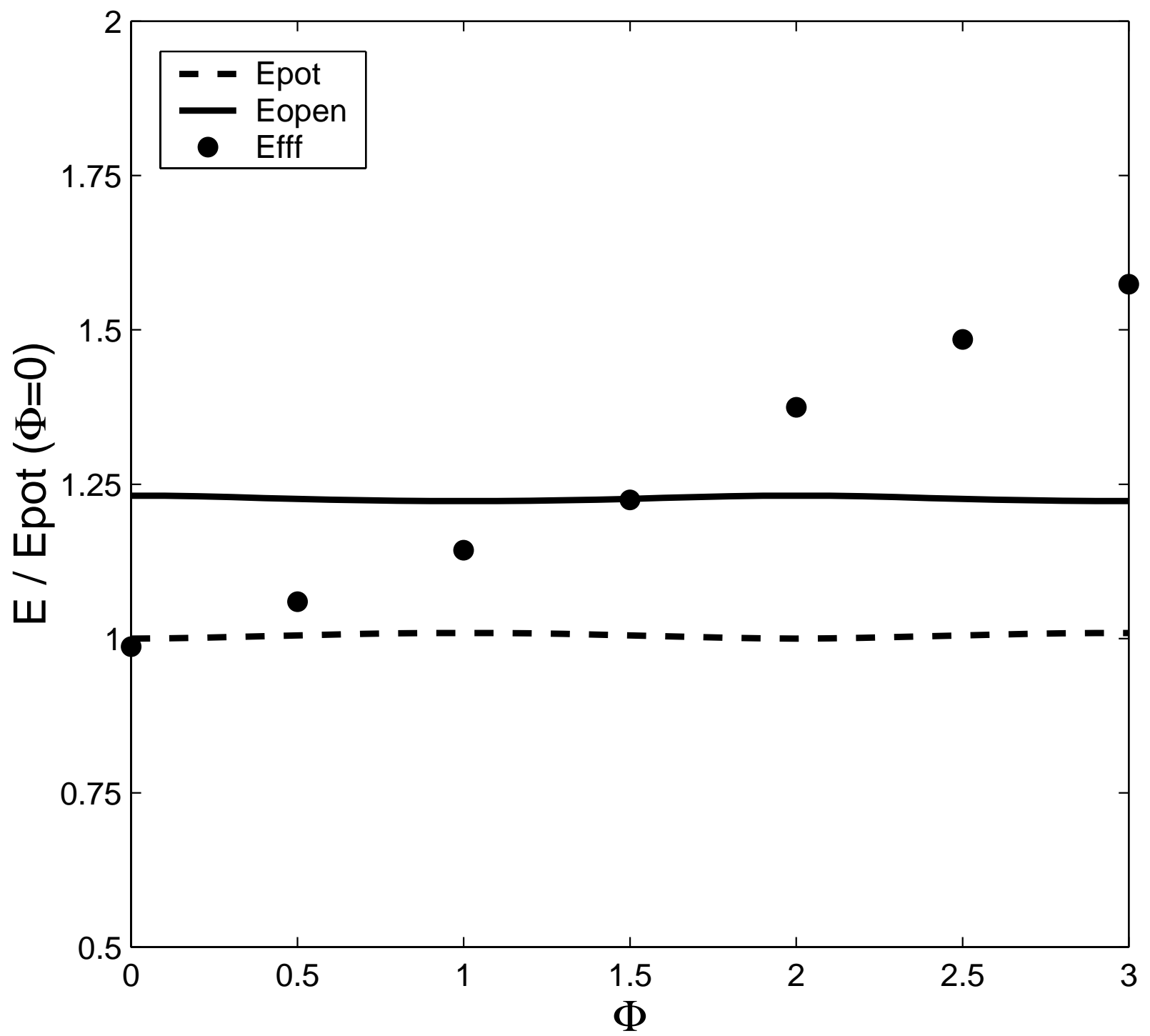

Fig. 3.- Energy of the numerically generated force-free fields for different twist angles. The energies of the potential fields (dashed curve) and of the open fields (solid curve) are also shown as a function of the twist angle. 

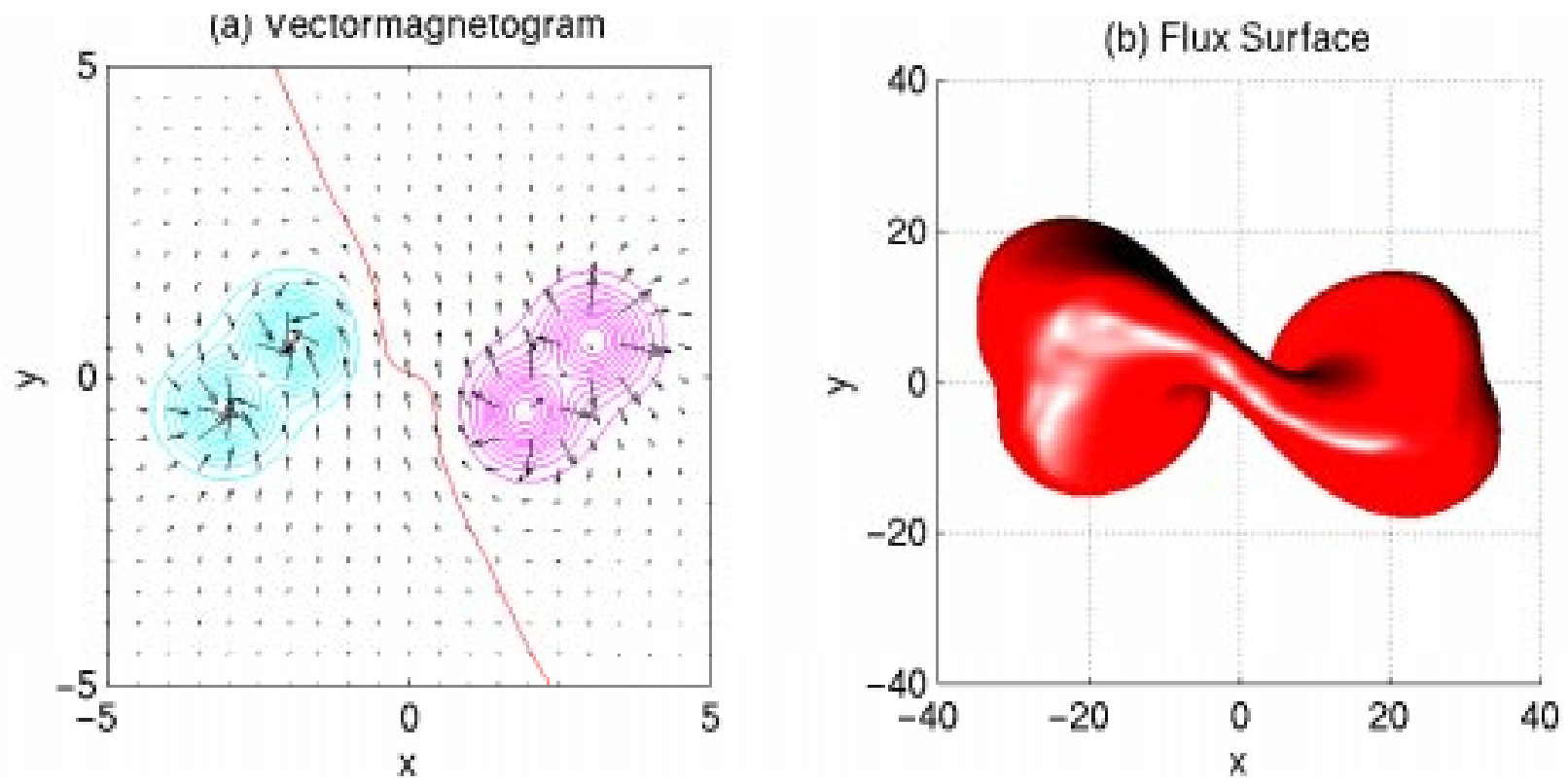

Fig. 4. - (a) A vectormagnetogram generated from our FFF solution for $\Phi=2.5 \pi$ at the height $z=0.22$. (b) The projected image of the flux surface containing $95 \%$ of the total flux of the inner flux tube (the red one in Figures 1 and 2). 


\section{External Distribution}

Plasma Research Laboratory, Australian National University, Australia

Professor I.R. J ones, Flinders University, Australia

Professor J oão Canalle, Instituto de Fisica DEQ/IF - UERJ , Brazil

Mr. Gerson O. Ludwig, Instituto Nacional de Pesquisas, Brazil

Dr. P.H. Sakanaka, Instituto Fisica, Brazil

The Librarian, Culham Laboratory, England

Library, R61, Rutherford Appleton Laboratory, England

Mrs. S.A. Hutchinson, JET Library, England

Professor M.N. Bussac, Ecole Polytechnique, France

Librarian, Max-Planck-Institut für Plasmaphysik, Germany

J olan Moldvai, Reports Library, MTA KFKI-ATKI, Hungary

Dr. P. Kaw, Institute for Plasma Research, India

Ms. P.J . Pathak, Librarian, Insitute for Plasma Research, India

Ms. Clelia De Palo, Associazione EURATOM-ENEA, I taly

Dr. G. Grosso, Instituto di Fisica del Plasma, Italy

Librarian, Naka Fusion Research Establishment, J AERI, J apan

Library, Plasma Physics Laboratory, Kyoto University, J apan

Research Information Center, National Institute for Fusion Science, J apan

Dr. O. Mitarai, Kyushu Tokai University, J apan

Library, Academia Sinica, Institute of Plasma Physics, People's Republic of China

Shih-Tung Tsai, Institute of Physics, Chinese Academy of Sciences, People's Republic of China

Dr. S. Mirnov, TRINITI, Troitsk, Russian Federation, Russia

Dr. V.S. Strelkov, Kurchatov Institute, Russian Federation, Russia

Professor Peter Lukac, Katedra Fyziky Plazmy MFF UK, Mlynska dolina F-2, Komenskeho Univerzita, SK-842 15 Bratislava, Slovakia

Dr. G.S. Lee, Korea Basic Science Institute, South Korea

Mr. Dennis Bruggink, Fusion Library, University of Wisconsin, USA

Institute for Plasma Research, University of Maryland, USA

Librarian, Fusion Energy Division, Oak Ridge National Laboratory, USA

Librarian, Institute of Fusion Studies, University of Texas, USA

Librarian, Magnetic Fusion Program, Lawrence Livermore National Laboratory, USA

Library, General Atomics, USA

Plasma Physics Group, Fusion Energy Research Program, University of California at San Diego, USA

Plasma Physics Library, Columbia University, USA

Alkesh Punjabi, Center for Fusion Research and Training, Hampton University, USA

Dr. W.M. Stacey, Fusion Research Center, Georgia Institute of Technology, USA

Dr. J ohn Willis, U.S. Department of Energy, Office of Fusion Energy Sciences, USA

Mr. Paul H. Wright, Indianapolis, Indiana, USA 
The Princeton Plasma Physics Laboratory is operated by Princeton University under contract with the U.S. Department of Energy.

\author{
Information Services \\ Princeton Plasma Physics Laboratory \\ P.O. Box 451 \\ Princeton, NJ 08543
}

Phone: 609-243-2750

Fax: 609-243-2751

e-mail: pppl_info@pppl.gov

Internet Address: http://www.pppl.gov 